\title{
Early Detection and Classification of Melanoma Skin Cancer
}

\author{
Dr.Abbas Hanon. Alasadi \\ Computer Science Department, Science College, Basrah University, Basrah, Iraq. \\ Email: Abbashh2002@yahoo.com \\ Baidaa M.ALsafy \\ Computer Science Department, Science College, Basrah University, Basrah, Iraq. \\ Email: baidaaalsafy@yahoo.com
}

\begin{abstract}
Melanoma is a form of cancer that begins in melanocytes (cells that make the pigment melanin). It can affect the skin only, or it may spread to the organs and bones. It is less common, but more serious and aggressive than other types of skin cancer. Melanoma can be of benign or malignant. Malignant melanoma is the dangerous condition, while benign is not. In order to reduce the death rate due to malignant melanoma skin cancer, it is necessary to diagnose it at an early stage.

In this paper, a detection system has been designed for diagnosing melanoma in early stages by using digital image processing techniques. The system consists of two phases: the first phase detects whether the pigmented skin lesion is malignant or benign; the second phase recognizes malignant melanoma skin cancer types. Both first and second phases have several stages. The experimental results are acceptable.
\end{abstract}

Index Terms-Melanoma; preprocessing; skin lesion segmentation; feature extraction; diagnosis.

\section{INTRODUCTION}

The skin anatomy has two main layers: epidermis (the outer layer) and dermis (the inner layer).The epidermis is made up of flat, scale-like cells called Squamous cells, and round cells called basal cells. The lower part of the epidermis contains melanocytes. Melanocytes are pigment cells that are found in the lower part of the epidermis and produce melanin, the pigment that gives skin its natural color [1]. When the skin is exposed to the sun, melanocytes produce more pigment, causing the skin to be bronzed, or darken which cause melanoma. Melanoma can be of Benign or Malignant [2] see Fig. 1.

The abnormal growths of melanocytes cause malignant melanoma cancer which invades or spreads to other parts of the body without normal controls, Malignant melanoma divided into Superficial Spreading Melanoma (constitutes about $75 \%$ of all non-melanomas), Nodular Melanoma (constitutes about $15 \%$ of all nonmelanomas), Lentigo malignant Melanoma (constitutes about $10 \%$ of all non-melanomas) and Acral Lentiginous Melanoma (constitutes about 5\% of all melanomas) [3] see Fig. 2.

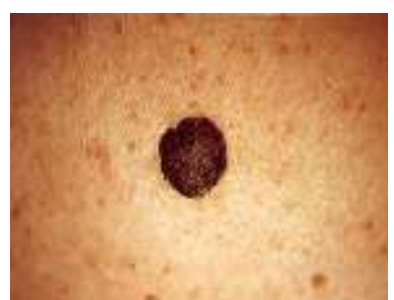

(a) (b)

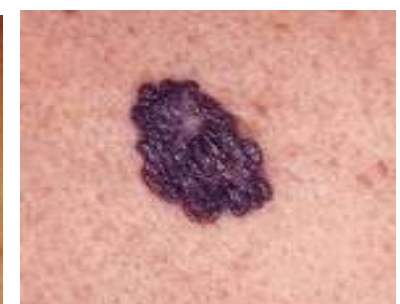

Fig. 1. Melanoma. (a) Benign. (b) Malignant.

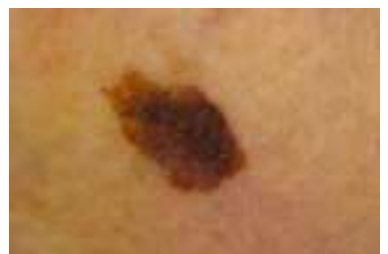

(a)

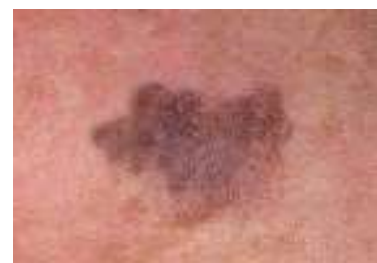

(c)

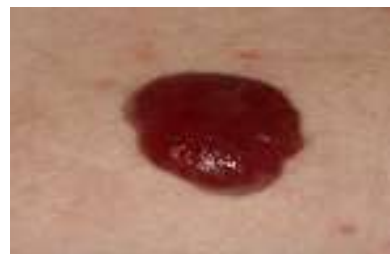

(b)

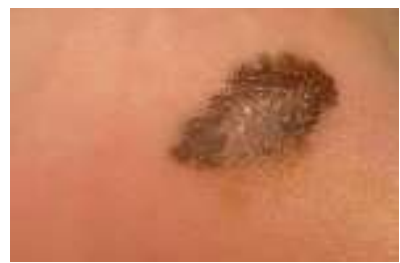

(d)
Fig. 2. Malignant Melanoma Types. (a) Superficial Spreading Melanoma. (b) Nodular Melanoma (c) Lentigo malignant Melanoma. (d) Acral Lentiginous Melanoma.

In order to reduce the death rate due to malignant melanoma skin cancer, it is necessary to diagnose it at an early stage. This paper proposes a system for diagnoses malignant melanoma in early stages.

\section{THE PRoposed ALGORITHMS}

This section gives a general view of the proposed algorithm for diagnosis melanoma skin cancer. The structure of the proposed system is shown in Fig. 3.

First stage in the skin cancer detection system is the input image. Image in digital format is given as input to the system. Next stage is preprocessing which includes denoising and hairs removal. The noises are denoised by Median Filtering; whilst, the hairs are removed by Dull 
Razor filter [5]. After filtering, the image is subjected to segmentation. Segmentation separates the suspicious lesion from normal skin. There are some unique features that distinguish the benign from malignant melanoma. The feature extraction techniques used here are variance and standard deviation for color features; Tamura method [10] for texture features; calculates regularity, symmetry, and diameter for shape features. The selected features are given as the input to Neural Network classifier (NN). The classifier classifies the given datasets into benign and malignant melanoma. Then, take malignant melanoma to distinguish between types. There are some unique features that distinguish the malignant melanoma types. Those features are extracted using feature extraction techniques. The feature extraction techniques used here are color moment descriptor for color features, GLCM method [12] for texture features, geometry features. The selected features are given as the input to $\mathrm{NN}$ classifier. The classifier classifies the given datasets into malignant melanoma types.

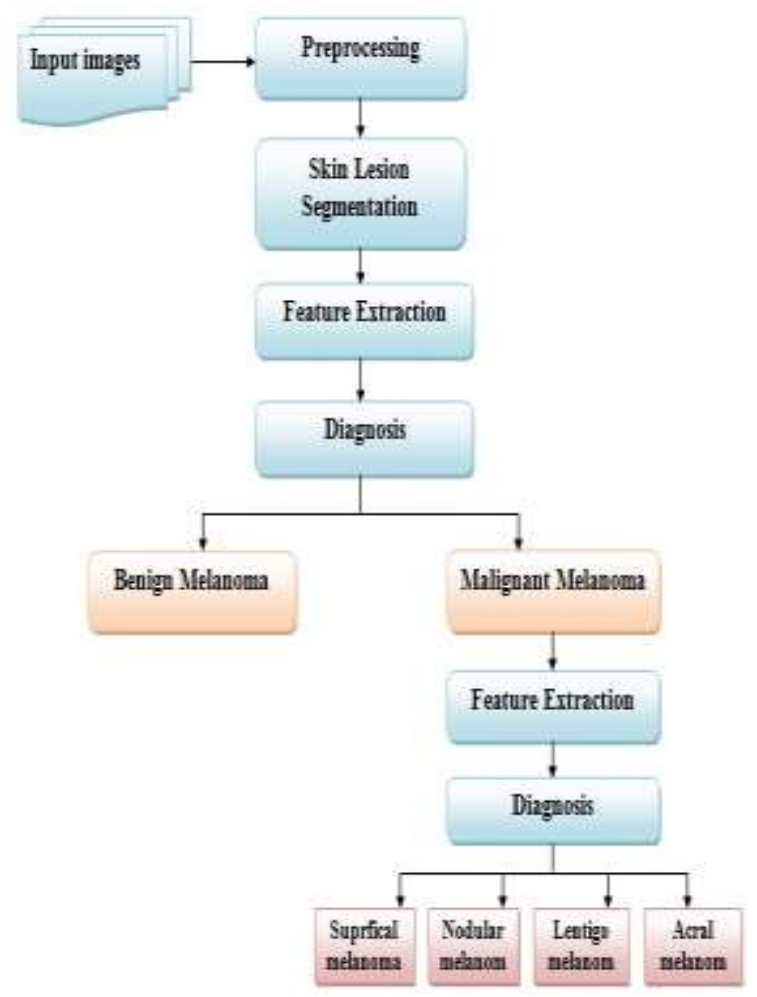

Fig. 3. The essential steps of the proposed system.

\subsection{Detecting Melanoma Lesions}

In the paper, detecting melanoma lesions involves four steps: preprocessing, lesion segmentation (separate lesion from skin), feature extraction (color, texture and shape features), and diagnosis by using neural network to detect if the lesion is benign or malignant melanoma. The images databases of melanoma skin cancer that have been adopted in this paper are obtained from the Lloyd Dermatology and Laser Center [4]. The database contains about 200 color images for melanoma skin cancer, images are captured in the JPEG format, with maximum resolution size $481 \times 734$ pixels, which were later resized to $256 \times 512$ pixels.

\subsubsection{Preprocessing}

The aim of pre-processing step is to enhance the images and remove unwanted effects. It involves three main processes:

\section{A. Hair Removal}

Hair removal is done by using Dull Razor filter for color images. Dull Razor filter is used by [5], which replaced hair pixels by neighboring pixels. Algorithm (1) describes the main steps of Dull Razor filter. Fig. 4 shows the result of Algorithm (1).

\section{Algorithm (1): Dull Razor}

Input: color images with hair.

Output: color images without hair.

Step1: It identifies the dark hair locations by a generalized gray-scale morphological closing operation.

step2: It verifies the shape of the hair pixels as thin and long structure, and replaces the verified pixels by a bilinear interpolation.

step3: It smoothes the replaced hair pixels with an adaptive median filter.

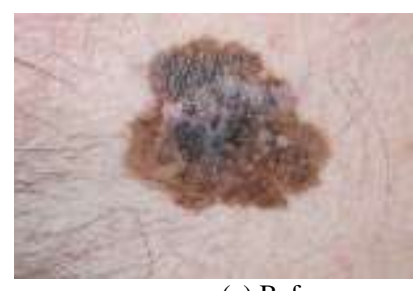

(a) Before

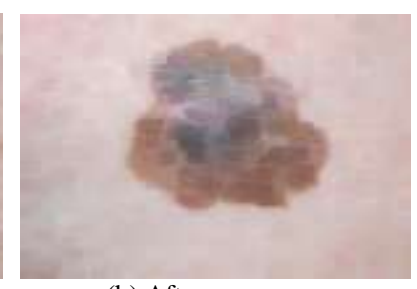

(b) After
Fig. 4. Dull Razor Filter.

\section{B. Noises Removal}

Even after hair removal, there may be some noises present in the image. Air bubbles, scratches in the skin etc. constitutes the noises. These noises are removed by using Median filtering proposed in [6]. Median Filtering is an image filtering method in which each pixel value in an image is replaced with the median value of its neighboring pixels including itself. Median filtering is used for minimizing the influence of small structures like thin hairs and isolated islands of pixels like small air bubbles see Fig. 5.

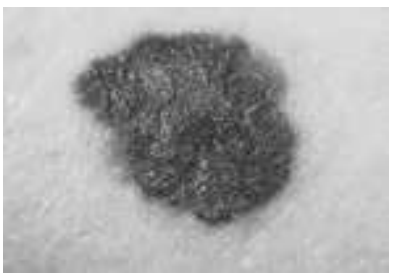

(a) Before

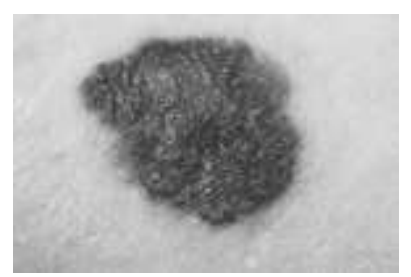

(b) After
Fig. 5. Noise removal. 


\section{Contrast Enhancement}

In this step, we are trying to increase the image clarity, obtain better performance and get high contrast. We use image adjustment which removes the unwanted part of the image that came from some noises (converted from negative to normal ones). It is done to enhance the shape and edges of image. In addition, contrast enhancement can sharpen the image border and improve the accuracy for segmentation, see Fig. 6.

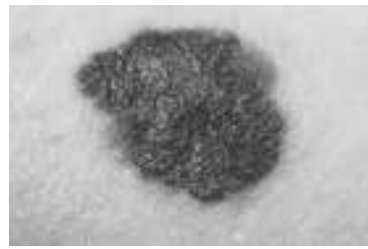

(a) Before

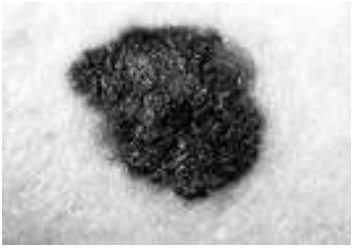

(b) After
Fig. 6. Contrast enhancement.

\subsubsection{Skin Lesion Segmentation}

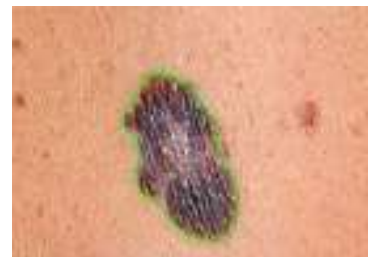

(a)

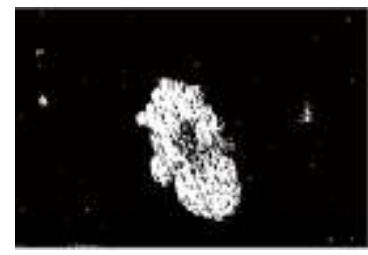

(c)

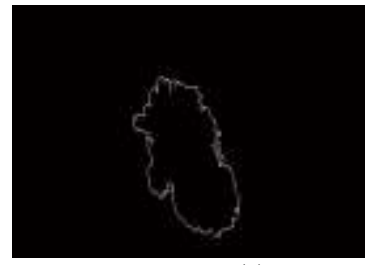

(e)

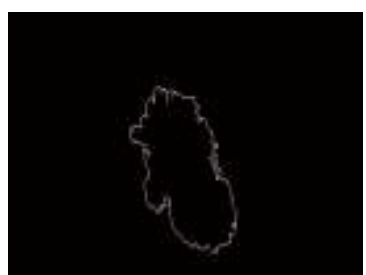

(g)

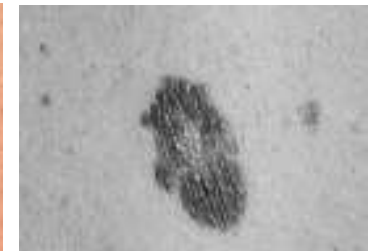

(b)

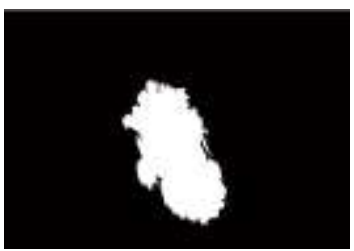

(d)

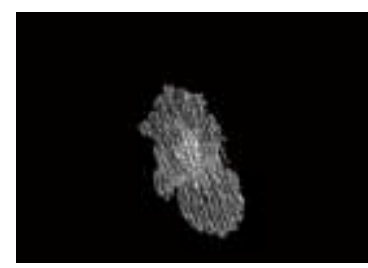

(f)

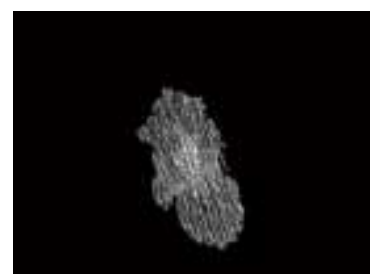

(h)
Fig. 7. The main lesion segmentation steps. (a) Original image. (b) Traced lesion (c) Image after preprocessing. (d) Applying thresholding and converting to binary image. (e) Remove unwanted pixels. (f) Apply morphological operation. (g) Detect border. (h) Lesion segmentation.

Actually, the segmentation step is an intermediate step to isolate the object of interest from the background, in skin lesion segmentation separates the suspicious lesion from normal skin. In this paper, the main steps for lesion segmentation can be categorized into four section (1) thresholding (2) morphological operation (3) border detection (4) lesion extraction. Fig. 7 shows the results of segmentation step. Algorithm (2) describes the steps of lesion segmentation algorithm.

\section{Algorithm (2): Lesion segmentation}

Input: All images after preprocessing step.

Output: Gray level images contain lesion only.

Step1: All the gray level images are of the size $(256 * 512)$, determine the number of train image is 100 images and stored all them in cell array.

Step2: Thresholding was used, the pixels of image less than 100 equals to zero.

Step3: Creates a structuring element with disk as shape and 4 as a radius.

Step4: Create Binary image with some unwanted pixels.

Step5: Remove unwanted pixels from binary image and producing another binary image without unwanted pixels (400 as unwanted pixels).

Step6: Clear the image border.

Step7: Fills holes in the binary image. A hole is a set of background pixels that cannot be reached by filling in the background from the edge of the image. By using Morphological operation.

Step8: Repeat step5 with (200 as unwanted pixels).

Step9: Performs morphological closing with the structuring element on the binary image. The morphological close operation is a dilation followed by erosion, using the same structuring element for both operations.

Step10: Repeat step7. The output is binary image.

Step11: Detect border of object by using canny edge detection to extract the edges and boundaries from image.

Step12: Convert the input gray level image (array) into unsigned 8-bit (1-byte) integers of class uint8.

Step13: Convert the image that results of the step10 (array) into unsigned 8-bit (1-byte) integers of class uint8.

Step14: Multiplies each element in array (image results from step 12) by the corresponding element in array (image results from step 13) and returns the product in the corresponding element of the output array.

\subsubsection{Feature Extraction}

There are some unique features that distinguish malignant melanoma from benign melanoma. These features are selected for classification purpose. The final result of features extraction task is a vector of features. Three types of feature are extracted; color, shape, and texture.

\subsubsection{Color Features}


One of the early signs of melanoma is the skin color variations. They are often colorful around brown, or black, depending on the production of the melanin pigment at different depth in the skin. The descriptors of color are mainly statistical parameters calculated from different color space channels, like variance and standard deviation of RGB or HSV. In this paper, color variance of RGB image has been calculated using HSV channel. Algorithm (3) describes the steps of color features algorithm.

\section{Algorithm (3): color features}

Input : color images for malignant and benign melanoma after trace and detect the lesion.

Output: color features vector.

Step1: convert the RGB color map into an HSV color map. The column of the output matrix represents Hue, Saturation, and Value respectively.

Step2: compute variance for each $\mathrm{H}, \mathrm{S}$, and V channel.

Step3: compute standard derivation for each $\mathrm{H}, \mathrm{S}$, and V channel.

Step4: The result from this algorithm is vector with sex values.

\subsubsection{Shape Features}

The shape features are asymmetry index, irregularity index, and diameter that calculate from lesion in binary image.

\section{A. Asymmetry}

Asymmetry is an important aspect of shape understanding is symmetry, which is very useful in pattern analysis. For a symmetric pattern, one needs only one half of the pattern with the axis of symmetry. If a part of the pattern is missing or noisy, with the help of symmetry one can complete the pattern or rid the pattern of noisy. Malignant skin lesions tend to have symmetric shape but benign lesions tend to have asymmetric shape. If the shape is completely symmetrical, the ratio is 1 . As the asymmetry increases, the ratio approaches closer to 0 .

Asymmetry Index is computed with the following equation [1].

$$
\mathrm{AI}=\frac{\Delta \mathrm{A}}{\mathrm{A}} \times 100
$$

Where, $\mathrm{A}$ is the area of the total Image. $\Delta \mathrm{A}$ is the area of difference between total image and lesion area.

\section{B. Irregularity}

Malignant skin lesions tend to have irregular borders with sharp edges and notches. Benign lesions tend to have smooth borders. If the shape is completely symmetrical, the ratio is 1 , else the ratio approaches closer to 0 . Irregularity index is a function of area (A) and perimeter $(\mathrm{P})$, calculated with the following equation [2]:

$$
\mathrm{IR}=\frac{4 \pi \mathrm{A}}{\mathrm{P}^{2}}
$$

For a perfect circle, the irregularity index is 1 . As the border becomes more irregular, the index reaches 0 .

\section{Diameter}

Malignant melanoma tends to grow larger than benign melanoma, and especially the diameter of $6 \mathrm{~mm}$, to find the diameter, calculates the distance between each pair of points. The maximum of these distances is the diameter. Algorithm (4) describes the steps of shape features algorithm.

\section{Algorithm (4): computes the shape features.}

Input: Binary image which results from segmentation step after apply morphological operation.

Output: shape features vector.

Step1: Compute the Area of the total Image.

Step2: Compute the lesion area.

Step3: Compute the Area difference between total image and lesion area.

Step4: Compute asymmetry index by using the equation (1).

Step5: Traces the exterior boundaries of objects, as well as boundaries of holes inside these objects, in the binary image.

Step6: Compute the pixels of the border.

Step7: Compute the perimeter of the Lesion.

Step8: Compute the area of the Lesion.

Step9: Compute border irregularity index by using the equation (2).

Step10: Determine the coordinates $(x, y)$ of each pixel of the lesion perimeter.

Step11: Calculate the distance between each pair of points.

Step12: The maximum of these distances is the diameter.

Step13: The result from this algorithm is a vector of features, which has three elements asymmetry, regularity and diameter.

\subsubsection{Texture Features}

The texture of an image region is determined by the way the gray levels are distributed over the pixels in the region. Texture features extract from gray level lesion image. In this paper, Tamura method is used to extract texture features.

Tamura method is an approach of devising texture features that correspond to human visual perception [10]. It defined six textural features (coarseness, contrast, directionality, line-likeness, regularity and roughness) and compared them with psychological measurements for human subjects. The first three features are achieved very successful results and are used in our evaluation, bothseparately and as joint values.

Coarseness aims to identify the largest size at which a 
texture exists, even where a smaller micro texture exists. Computationally one first takes average at every point over neighborhoods the linear size of which are powers of 2 . The average over the neighborhood of size $2 k \times 2 k$ at the point $(x, y)$ is

$$
A_{k}(x, y)=\sum_{i=x-2^{k-1}}^{x+2^{k-1}-1 y-2^{k-1}} \sum^{k-1}-1
$$

Contrast aims to capture the dynamic range of grey levels in an image, together with the polarization of the distribution of black and white. The first is measured using the standard deviation of grey levels and the second the kurtosis $\alpha 4$. The contrast measure is therefore defined as:

$$
u_{i}=\frac{1}{N} \sum_{J=1}^{N} f_{i j}
$$

Where $\alpha_{4}=\mu_{4} / \sigma^{4}, u_{4}$ is the fourth moment about the mean and $\sigma$ is the variance

Directionality is a global texture property. Patterns can be highly directional (e.g., a brick wall) or may be nondirectional, as in the case of a picture of a cloud. The degree of directionality, measured on a scale of 0 to 1 , can be used as a descriptor. Algorithm (5) describes the steps of texture features algorithm.

\section{Algorithm (5): compute texture feature}

Input: Gray level image which results from the last step of lesion segmentation 0

Output: Texture features vector.

Step1: Resize all the image of gait cycle to size $128 *$ 128.

Step2: Compute the texture features to Tamura coarseness, contrast and directionality as in the equations (3) and (4).

Step3: The result from this algorithm is a vector with three values.

\subsubsection{Diagnosis}

The objective of the step is to distinguish between malignant and benign melanoma. In this paper, neural network is used for diagnoses between malignant and benign melanoma. Algorithm (6) describes the steps of neural network algorithm.

\section{Algorithm (6): NN Classification}

Input : A vector of features

Output: Total accuracy from the NN.

Step1: Create feed forward neural network with two hidden layer, 150,100 neurons in each hidden layer, input layer for neural network is determined by characteristics of inputs. We have twelve attribute features vector. Therefore, number of neuron in input layer is twelve, and output layer neuron determined by number of class, we have two classes (malignant and benign) therefore the number of neuron in output layer are two.

Step2: Determined the important parameter, learning rate equal to 0.00001 , epochs equal to 20000 , maximum number of iterations, training time infinity, data division function (divide rand), transfer function of $i^{\text {th }}$ layer hyperbolic tangent sigmoid transfer function is used 'tansig', linear activation function is selected for output layer 'purelin', performance function, default = 'mse 'and training function is back propagation function (Scaled conjugate gradient), weight and bias is generating randomly.

Step3: Train the network with train data and target matrix (target matrix is a matrix with two rows and two columns each row consists of a vector of all zero values except for a 1 in element $i$, where $i$ is the class they are to represent).

Step4: Simulates the neural network by taking the initialized net and network input matrix (train data), return the indices to the largest output as a class predict.

Step5: Compute the network performance.

Step6: Simulates the neural network by taking the training net and test data return the indices to the largest output as class predict.

Step7: Compute the network performance.

\subsection{Detecting Malignant Melanoma}

Detecting malignant melanoma types involves two steps: feature extraction, and neural network based classification. We take the malignant melanoma images only that result from the first phase to extract features and diagnoses malignant melanoma types.

\subsubsection{Feature Extraction}

These features are selected for distinguish malignant melanoma types. Three feature extraction techniques are proposed here; color, geometry and texture features.

\subsubsection{Color Features}

The color features are mean, skewness, and standard derivation. These features extract using Color Moment (CM) descriptor proposed in [11,12]. The common moments are mean, standard deviation and skewness; the corresponding calculation can be defined as follows respectively:

$$
\begin{gathered}
\mu_{i}=\frac{1}{N} \sum_{J=1}^{N} f_{i J} \\
\sigma_{i}=\left(\frac{1}{N} \sum_{J=1}^{N}\left(f_{i J}-\mu_{i}\right)^{2}\right)^{\frac{1}{2}}
\end{gathered}
$$




$$
\gamma_{i}=\left(\frac{1}{N} \sum_{J=1}^{N}\left(f_{i J}-\mu_{i}\right)^{3}\right)^{\frac{1}{3}}
$$

Where $f_{i j}$ is the color value of the $\mathrm{i}^{\text {th }}$ color component of the $\mathrm{j}^{\text {th }}$ image pixel and $N$ is the total number of pixels in the image. $\mu_{i}, \sigma_{i}, \gamma_{i}(i=1,2,3)$ denote the mean, standard deviation, and skewness of each channel of an image respectively. Algorithm (6) describes the steps of color features extraction algorithm.

\section{Algorithm (6): color features extraction}

Input: Color image for malignant melanoma.

Output: Color features vector.

Step1: Convert the RGB color map into an HSV color map. The column of the output matrix represents Hue, Saturation, and Value respectively.

Step2: Compute mean for each H, S, and V channel as in the equations (5).

Step3: Compute standard derivation for each $\mathrm{H}, \mathrm{S}$, and $\mathrm{V}$ channel as in the equations (6).

Step4: Compute skewness for each H, S, and V channel as in the equations (7).

Step5: The result from this algorithm is vector with nine values.

\subsubsection{Geometry Features}

The geometry features include Area of the lesion, Perimeter-to-Area Ratio of the lesion and, Eccentricity. We divide the perimeter by area using the hypothesis that a lesion with a large perimeter-to-area ratio will likely have a jagged boundary, while a pigmented lesion with a small perimeter-to-area ratio will likely have an edge that is smooth. Eccentricity is defined as the ratio of the Eigen values of the covariance matrix that corresponds to a binary image of the shape. To calculate eccentricity, we use the eccentricity argument from the MATLAB function regionprops, which determines the ratio of the distance between the foci of the ellipse fitted to the shape and its major axis length. The output is between 0 and 1 , with 0 representing a circle and 1 a line. Algorithm (7) describes the steps of geometry features algorithm.

\section{Algorithm (7): compute geometry features.}

Input: Binary image which results from segmentation step after apply morphological operation.

Output: Geometry features vector

Step1: Compute the area of the lesion from the MATLAB function regionprops.

Step2: Compute the Perimeter of the lesion from the MATLAB function regionprops.

Step3: Compute the Perimeter-to-Area Ratio by divided the perimeter to area.

Step4: Compute the Eccentricity from the MATLAB function regionprops.

Step5: The result from this algorithm is vector with three values.

\subsubsection{Texture Features}

The texture features consist of contrast, correlation, energy, and homogeneity. All are computed from GLCM proposed in [13] for the four directions $(0,45,90$, and135)) to gray level image of malignant melanoma. These features are described as below:

Contrast measures the amount of local variations in an image.

$$
\text { Contrast }=\sum_{i} \sum_{j}(i-j)^{2} P(i, j)
$$

Correlation is a measurement of gray tone linear dependencies in the image.

$$
\text { Correlation }=\frac{\left.\left\{\sum_{i} \sum_{j}(i j) P(i, j)\right\}-\mu_{x} \mu_{y}\right\}}{\sigma_{x} \sigma_{x}}
$$

Energy is a measurement of texture uniformity of an image, the more homogeneous the image, the larger the value.

$$
\text { Energy }=\sum_{i} \sum_{j} P(i, j)^{2}
$$

Homogeneity is a measurement of the amount of local uniformity present in the image

$$
\text { Homogeneity }=\sum_{i} \sum_{j} \frac{P(i, j)}{1+|i-j|}
$$

Algorithm (8) used to extract texture feature from gray level Co-occurrence matrix for gray level image

\section{Algorithm (8): compute texture feature.}

Input: Gray level image which results from the last step of lesion segmentation.

Output: Texture features vector

Step1: Resize the entire image to size $128 * 128$.

Step2: Compute gray level co-occurrence matrix to the gray level image with four off set ([0 1 1$],\left[\begin{array}{ll}-1 & 0\end{array}\right]$, $\left.\left[\begin{array}{ll}-1 & 1\end{array}\right],\left[\begin{array}{ll}-1 & -1\end{array}\right]\right)$. The GLCM creates by calculating how often a pixel with gray-level (grayscale intensity) value $i$ occurs horizontally adjacent to a pixel with the value $j$. (You can specify other pixel spatial relationships using the 'Offsets' parameter ([0 1],[-1 0],[-1 1],[-1 1]) Each element $(i, j)$ in GLCM specifies the number of times that the pixel with value $i$ occurred horizontally adjacent to a pixel with value $j$. 
Step3: Compute the texture feature to GLCM contrast, correlation, energy, homogeneity.

Step4: The result from this algorithm is vector with sixteen values.

After extract the three types of features malignant melanoma images, combine in one vector to use in the diagnosis step to recognize between malignant melanoma types, you must refer that features extraction step make in training and testing phases.

\subsubsection{Diagnosis}

The last step in the second phase from the proposed system is diagnosis, distinguish between malignant melanoma types, after obtained features vector and store it ,the diagnosis step is followed, in this paper, we use neural network to diagnoses between malignant melanoma types.

\section{Algorithm (6): NN Classification Algorithm}

Output: Total accuracy from the NN.

Step1: Create feed forward neural network with five hidden layer, number of neuron in each hidden layer 150, $100,150,100,100$ respectively, input layer for created neural network is determined by characteristics of inputs. We have twenty-eight attribute features vector. Therefore, number of neuron in input layer is twenty eight, and out layer neuron determined by number of class, we have four classes (malignant melanoma types) there for the number of neuron in output layer are four.

Step2: Determined the important parameter, learning rate equal to 0.00001 , epochs equal to 20000 , maximum number of iterations, training time infinity, data division function (divide rand), transfer function of $i^{\text {th }}$ layer hyperbolic tangent sigmoid transfer function is used 'tansig', linear activation function is selected for output layer 'purelin', performance function, default $=$ 'mse 'and training function is back propagation function (Scaled conjugate gradient), weight and bias is generating randomly.

Step3: Train the network with train data and target matrix (target matrix is matrix with four row and four column each row consist of vector of all zero values except for a 1 in element $i$, where $i$ is the class they are to represent).

Step4: Simulates the neural network by taking the initialized net and network input matrix (train data), return the indices to the largest output as class predict.

Step5: Compute the network performance.

Step6: Simulates the neural network by taking the training net and test data, return the indices to the largest output as class predict.

Step7: Compute the network performance.

\section{EXPERIMENT RESULTS AND CONCLUSIONS}

Early melanoma skin cancer diagnostic system using computer based techniques is more efficient than the conventional Biopsy methods. The cost involved as well as the time taken for detection is less in this proposed methodology. The methodology incorporates artificial intelligence and digital image processing for skin cancer detection. Neural Network based classifier proved to be very efficient in decision making as well as pattern recognition applications. The proposed method has an accuracy of $98 \%$ for recognition melanoma skin cancer (benign \& malignant) and $93 \%$ for recognition malignant melanoma types, which is much higher than that of conventional methods.

Table 1 illustrates the classification results of NN for melanoma skin cancer. Table 2 shows the recognition rate of malignant melanoma types.

Table 1. Classification Result from NN for melanoma skin cancer

\begin{tabular}{|c|c|c|c|}
\hline \multirow{2}{*}{ Data type } & Feature type & \multicolumn{2}{|c|}{$\begin{array}{c}\text { Classification } \\
\text { Accuracy } \%\end{array}$} \\
\cline { 2 - 4 } & $\begin{array}{c}\text { Train } \\
\text { Data }\end{array}$ & $\begin{array}{c}\text { Test } \\
\text { Data }\end{array}$ \\
\hline \multirow{4}{*}{$\begin{array}{c}\text { Melanoma Skin } \\
\text { Cancer } \\
\text { (Benign \& } \\
\text { Malignant) }\end{array}$} & Color Features & 100 & 50 \\
\cline { 2 - 4 } & $\begin{array}{c}\text { Shape Features } \\
\text { Texture Features }\end{array}$ & 100 & 50 \\
\cline { 2 - 4 } & $\begin{array}{c}\text { Color \& Shape } \\
\text { Features }\end{array}$ & 100 & 50 \\
\cline { 2 - 4 } & $\begin{array}{c}\text { Color \& Texture } \\
\text { Features }\end{array}$ & 100 & 50 \\
\cline { 2 - 4 } & $\begin{array}{c}\text { Color, Shape and } \\
\text { Texture Features }\end{array}$ & 100 & 98 \\
\hline \multirow{2}{*}{ Total accuracy from the NN } & \multicolumn{2}{|c}{$98 \%$} \\
\hline
\end{tabular}

Table 2. Classification Result from NN for malignant melanoma types

\begin{tabular}{|c|c|c|c|}
\hline \multirow{2}{*}{ Data type } & \multirow{2}{*}{ Feature type } & \multicolumn{2}{|c|}{$\begin{array}{l}\text { Classification } \\
\text { Accuracy } \%\end{array}$} \\
\hline & & $\begin{array}{l}\text { Train } \\
\text { Data }\end{array}$ & $\begin{array}{l}\text { Test } \\
\text { Data }\end{array}$ \\
\hline \multirow{7}{*}{$\begin{array}{c}\text { Malignant } \\
\text { Melanoma } \\
\text { Types }\end{array}$} & Color Features & 100 & 25 \\
\hline & Geometry Features & 100 & 43 \\
\hline & Texture Features & 100 & 20 \\
\hline & $\begin{array}{c}\text { Texture \& Geometry } \\
\text { Features }\end{array}$ & 100 & 50 \\
\hline & $\begin{array}{l}\text { Color \& Texture } \\
\text { Features }\end{array}$ & 100 & 76 \\
\hline & $\begin{array}{l}\text { Color \& Geometry } \\
\text { Features }\end{array}$ & 100 & 75 \\
\hline & $\begin{array}{c}\text { Color, Geometry and } \\
\text { Texture Features } \\
\end{array}$ & 100 & 93 \\
\hline \multicolumn{2}{|c|}{ Total accuracy from the NN } & \multicolumn{2}{|c|}{$93 \%$} \\
\hline
\end{tabular}

\section{REFERENCES}

[1] National Cancer Institute, "Melanoma skin cancer ", U.S. Department of Health and Human Services, National Institutes of Health, 2010. 
[2] Aswin.R.B, J. Abdul Jaleel, Sibi Salim, "Implementation of ANN Classifier using MATLAB for Skin Cancer Detection", College of Engineering and Technology during December, 2013 at Trivandrum, Kerala, India.

[3] Bareqa Salah, Mohammad Alshraideh, Rasha Beidas and Ferial Hayajneh, "Skin Cancer Recognition by Using a Neuro-Fuzzy System', The University of Jordan, Amman 11942, Jordan, 2011.

[4] MELANOMA

DATABASE: http://www.lloydderm.com/searchresults.php?search=ima ges\&sort=score.

[5] T. Y. SATHEESHA, D. SATYANARAYANA and M. N. GIRIPRASAD,“A Pixel Interpolation Technique for curved hair removal in skin images to support melanoma detection", Journal of Theoretical and Applied Information Technology, 2005.

[6] Gajanand Gupta, “ Algorithm for Image Processing Using Improved Median Filter and Comparison of Mean, Median and Improved Median Filter", International Journal of Soft Computing and Engineering (IJSCE) ISSN 2231-2307, Volume-1, Issue-5, November 2011.

[7] Salem Saleh Al-amri, N.V. Kalyankar and Khamitkar S.D , "Image Segmentation by Using Thershod Techniques", JOURNAL OF COMPUTING, VOLUME 2 , ISSUE 5, MAY 2010, ISSN 2151-9617,2010.

[8] Md.Amran Hossen Bhuiyan, Ibrahim Azad, and Md.Kamal Uddin, "Image Processing for Skin Cancer Features Extraction", International Journal of Scientific \& Engineering Research Volume 4, Issue 2, February-2013.

[9] Nikhil Cheerla and Debbie Frazier," Automatic Melanoma Detection Using Multi-Stage Neural Networks", International Journal of Innovative Research in Science, Engineering and Technology, Vol. 3, Issue 2, February 2014.

[10] Peter Howarth and Stefan R"uger,' Evaluation of Texture Features for Content-Based Image Retrieval", Department of Computing, Imperial College London, South Kensington Campus, London, 2004.

[11] Dong ping Tian, "A Review on Image Feature Extraction and Representation Techniques", International Journal of Multimedia and Ubiquitous Engineering Vol. 8, No. 4, July, 2013.

[12] Mohammad Saber Iraji,Azam Tosinia, "Skin Color Segmentation in YCBCR Color Space with Adaptive Fuzzy Neural Network (Anfis) ”,IJIGSP,Vol.4,No.4,pp.35-41,2012.

[13] Fritz Albregtsen, "Statistical Texture Measures Computed from Gray Level Coocurrence Matrices", Image Processing Laboratory Department of Informatics University of Oslo November 5, 2008.

\section{Authors' Profiles}

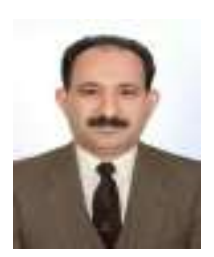

Abbas H. Hassin Alasadi is Assistant Professor and Postgraduate Program Coordinator of the Department of Computer Science at Basrah University. He received his $\mathrm{PhD}$ degree from School of Engineering and Computer Science / Harbin Institute of Technology, China. He spent more than ten years as Assistant Professor at different Universities abroad the current position. His research interests include Medical Image processing, Biometrics, Information retrieval, and Humancomputer interaction. His research work have been published in various international journals and conferences.
Dr. Abbas is an active reviewer in many journals of the areas of computer science and software engineering. He is one of ACIT members.

Baidaa M.ALsafy: MSc student at the Computer Science Department, Science College, Basrah University, Basrah, Iraq.

How to cite this paper: Abbas Hanon. Alasadi, Baidaa M.ALsafy,"Early Detection and Classification of Melanoma Skin Cancer", International Journal of Information Technology and Computer Science(IJITCS), vol.7, no.12, pp.67-74, 2015. DOI: $10.5815 /$ ijitcs.2015.12.08 